\title{
Reconstruction of Irregularly Sampled Discrete-Time Bandlimited Signals with Unknown Sampling Locations
}

\author{
Pina Marziliano and Martin Vetterli, Fellow IEEE
}

\begin{abstract}
The purpose of this paper is to develop methods that can reconstruct a bandlimited discrete-time signal from an irregular set of samples at unknown locations. We define a solution to the problem using first a geometric and then an algebraic point of view. We find the locations of the irregular set of samples by treating the problem as a combinatorial optimization problem. We employ an exhaustive method and two descent methods: the random search and cyclic coordinate methods. The numerical simulations were made on three types of irregular sets of locations: random sets; sets with jitter around a uniform set; and periodic nonuniform sets. Furthermore, for the periodic nonuniform set of locations, we develop a fast scheme that reduces the computational complexity of the problem by exploiting the periodic nonuniform structure of the sample locations in the DFT.
\end{abstract}

Index Terms-Bandlimited, irregular sampling, jitter, periodic nonuniform, unknown locations.

\section{INTRODUCTION}

$\mathbf{T}$ HE WELL-KNOWN sampling theorem states that a continuous-time bandlimited signal can be recovered from a uniform set of samples if the sampling rate is twice the bandlimit. The Kadec-1/4 theorem [1] is its equivalent when the samples are irregularly spaced. The irregular sampling problem for continuous and discrete-time bandlimited signals has been treated in [2]-[4] and for two-dimensional (2-D) signals in [5]. In all of these problems, the band limit, the sample values, and their locations are supposedly known or given. In practice, the sampling locations are not always known, for instance, when there is a certain jitter around a uniform set of locations as in Fig. 1(a) or as in Fig. 1(b), when there is a certain unknown shift in a periodic nonuniform set of samples. This is the motivation for studying the irregular sampling problem with unknown sampling locations [6]. The question we intend to answer is the following: How can we recover a discrete-time $L$ bandlimited signal of length $N$ from $K$ irregularly spaced sample values without knowing the sampling locations $(L \leq K<N)$ ?

\footnotetext{
Manuscript received December 22, 1999; revised August 9, 2000. The associate editor coordinating the review of this paper and approving it for publication was Dr. Xiang-Gen Xia.

P. Marziliano is with the Communication Systems Department, Swiss Federal Institute of Technology, Lausanne, Switzerland (e-mail: Pina.Marziliano@epfl.ch).

M. Vetterli is with the Communication Systems Department, Swiss Federal Institute of Technology, Lausanne, Switzerland and with the Electrical Engineering and Computer Science Department, University of California, Berkeley, CA 94720 (e-mail: Martin.Vetterli@epfl.ch).

Publisher Item Identifier S 1053-587X(00)10161-8.
}

In [7], a similar problem is studied in the context of error correction. The authors reconstruct a passband signal of length $N$ given a noisy signal with $n$ errors in unknown locations. To determine the locations of the errors, they require the knowledge of at least $2 n$ values in the error spectrum. In other words, they are looking for the complement set of the sampling locations given information on some part of the error spectrum. Our problem differs from theirs in that we have no knowledge of the error spectrum except its structure; we have only $K$ sample values, whereas they have the entire signal, although it is noisy; the number of samples $K$ is usually much larger than the number of errors $n$; therefore, our problem has more unknown variables.

The paper is organized as follows. In Section II, we formulate the irregular sampling with unknown locations (ISUL) problem for discrete-time bandlimited signals. We define a solution by describing the problem geometrically and algebraically. We also study the different conditions for which there may be multiple solutions and a unique solution. Three solving methods for the ISUL problem are described in Section III. The first is an exhaustive search method that tests all the possible sets of locations. The two other methods (the random search and cyclic coordinate method) are descent algorithms in which a solution set of locations is obtained by perturbing a location. The random search method differs from the cyclic coordinate method in that the perturbation follows a probability density function, whereas the latter is deterministic. In Section IV, we describe three experiments. In the first experiment, we consider signals where the irregular sampling set of locations is random, and the second is one where the irregular set is composed of a uniform set with a certain jitter. We show that the cyclic coordinate method is more successful in finding the sets of locations than the random search method. The third experiment involves reconstructing a signal where the sampling locations are formed by a union of uniform sets differing by shifts. The shifts are unknown. We determine these shifts using the exhaustive method. We will show that the size as well as the computational costs of the problem are reduced by exploiting the periodic structure in the data and in the DFT matrix.

The notations are as follows: Vectors and matrices are denoted by bold lowercase and capital letters, respectively. A finite discrete-time signal of length $N$ will be represented by a vector $\mathrm{x}=\mathrm{x}(\mathcal{N})=\left(x_{0}, x_{1}, \cdots, x_{N-1}\right)^{T}$, where $\mathcal{N}=$ $\{0,1, \cdots, N-1\}$ is the index set. Similarly, for matrices, suppose $\mathbf{F}$ is an $M \times N$ real or complex-valued matrix. Let $\alpha \subseteq\{0, \cdots, M-1\}$ and $\beta \subseteq\{0, \cdots, N-1\}$ be two index 


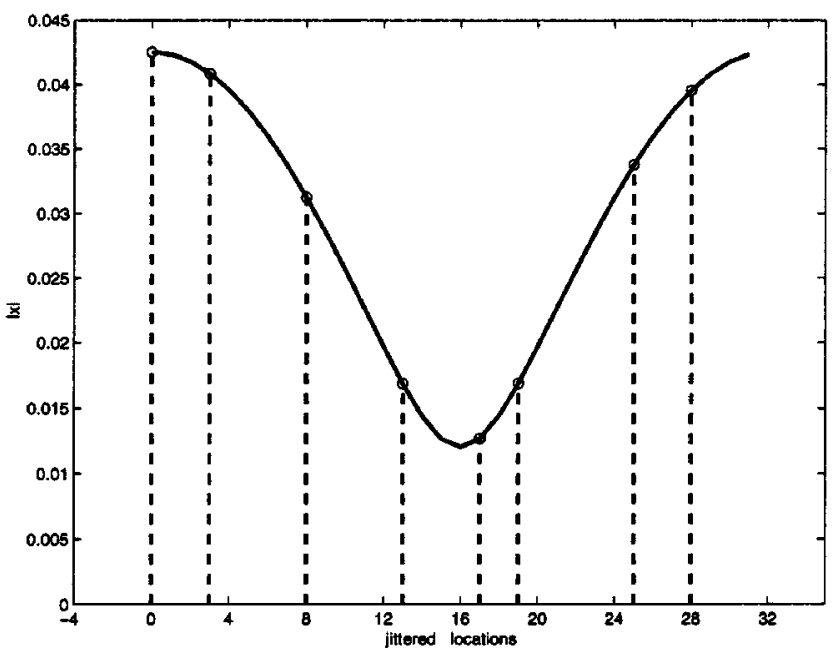

(a)

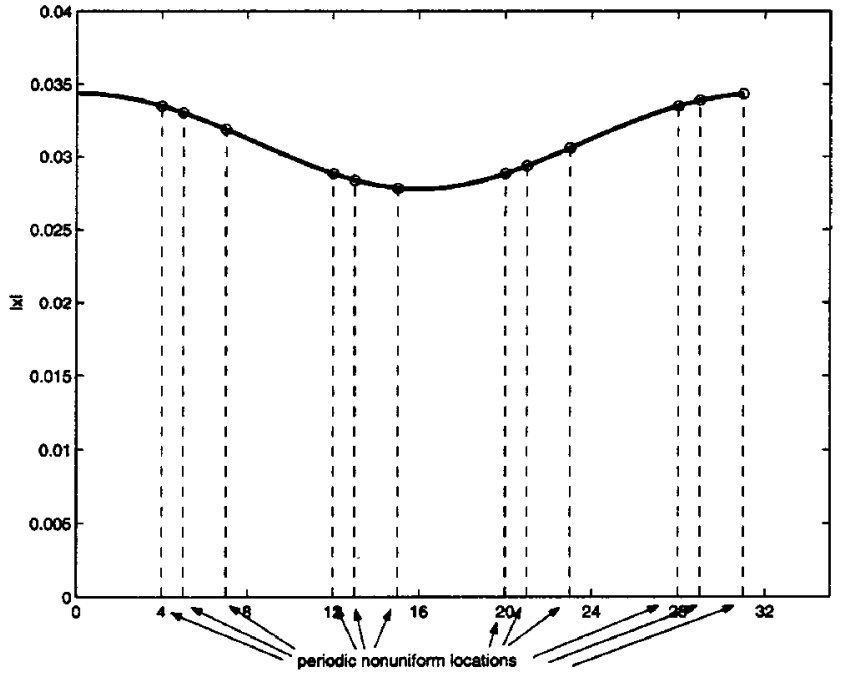

(b)

Fig. 1. (a) Discrete-time signal of length $N=32$ with a jitter of \pm 1 around $\{n T\}_{n=0}^{K-1}$, where $T=4$ and $K=8$. (b) Periodic nonuniformly sampled discrete-time signal of length $N=32$ composed of $C=3$ uniform sets of length $K_{0}=4$ with shifts $r=(4,5,7)$.

sets. Then, the submatrix $\mathbf{F}(\alpha, \beta)$ is composed of the rows of $\mathbf{F}$ indexed by $\alpha$ and columns of $\mathbf{F}$ indexed by $\beta$; see [8].

\section{PROBlem Formulation AND SOLUTION}

The ISUL problem consists of reconstructing a length- $N$ discrete-time $L-B L$ signal $\mathbf{x}=\left(x_{0}, x_{1}, \cdots, x_{N-1}\right)^{T}$ given $K$ sample values $\mathbf{s}=\mathbf{x}\left(\mathcal{N}_{K}\right)=\left(x_{n_{1}}, x_{n_{2}}, \cdots, x_{n_{K}}\right)^{T}$, where $\mathcal{N}_{K}=\left\{n_{i}\right\}_{i=1}^{K}$ is an unknown irregularly spaced ordered subset of $\mathcal{N}=\{0,1, \cdots, N-1\}, L \leq K<N$. We recall the definition of bandlimitedness for discrete-time signals.

Definition 1: A discrete-time signal $\mathrm{x}=\left(x_{0}, \cdots, x_{N-1}\right)^{T}$ of length $N$ is bandlimited to $L$ (in the low-pass sense) if the last $N-L$ components of the discrete Fourier transform $\hat{\mathrm{x}}$ are zero, i.e.,

$$
\begin{gathered}
\left(\begin{array}{c}
\hat{x}_{0} \\
\hat{x}_{1} \\
\vdots \\
\hat{x}_{L-1} \\
0 \\
\vdots \\
0
\end{array}\right)=\left[\begin{array}{cccc}
1 & 1 & \cdots & 1 \\
1 & W_{N} & \cdots & W_{N}^{N-1} \\
\vdots & \vdots & \cdots & \vdots \\
1 & W_{N}^{N-1} & \cdots & W_{N}^{(N-1)(N-1)}
\end{array}\right]\left(\begin{array}{c}
x_{0} \\
x_{1} \\
\vdots \\
x_{N-1}
\end{array}\right) \\
\hat{\mathbf{x}}=\mathbf{D F T}_{N} \cdot \mathbf{x}
\end{gathered}
$$

where DFT $_{N}$ is the discrete Fourier transform matrix, and $W_{N}=e^{-i 2 \pi / N}\left(W_{N}^{n}=W_{N}^{n \bmod N}\right)$. To solve the ISUL problem, we must have a clear understanding of the subspaces to which the irregular set of sample values s belong.

\section{A. Derivation of a Solution-Subspace and Algebraic Approach}

In this section, we derive a solution to the ISUL problem by describing it alternatively via a subspace and an algebraic ap- proach. We begin by analyzing the signal subspace followed by that of the irregular set of sample values.

From (1), we obtain an expression for the signal in terms of the spectrum by inverting the $\mathrm{DFT}_{N}$ matrix and noticing that the last $N-L$ columns do not contribute to the signal (since the last $N-L$ spectrum values are zero). We obtain

$$
\begin{aligned}
\left(\begin{array}{c}
x_{0} \\
x_{1} \\
\vdots \\
x_{N-1}
\end{array}\right)= & \frac{1}{N}\left[\begin{array}{cccc}
1 & 1 & \cdots & 1 \\
1 & W_{N}^{-1} & \cdots & W_{N}^{-(L-1)} \\
\vdots & \vdots & \cdots & \vdots \\
1 & W_{N}^{-(N-1)} & \cdots & W_{N}^{-(N-1)(L-1)}
\end{array}\right] \\
& \cdot\left(\begin{array}{c}
\hat{x}_{0} \\
\hat{x}_{1} \\
\vdots \\
\hat{x}_{L-1}
\end{array}\right)
\end{aligned}
$$

or using the notation described in Section I,

$$
\mathrm{x}(\mathcal{N})=\frac{1}{N} \mathbf{F}(\mathcal{N}, \mathcal{L}) \hat{\mathbf{x}}(\mathcal{L})
$$

where $\mathbf{F}$ is the conjugate transpose of the $\mathbf{D F T}_{N}$ [since $\left.\left(\mathbf{D F T}_{N}\right)^{-1}=(1 / N) \mathbf{D F T}_{N}^{*}\right]$, and $\mathcal{L}=\{0,1, \cdots, L-1\}$. Expanding (2), we see that $\mathbf{x}(\mathcal{N})$ is a linear combination of the $L$ first columns of $\mathbf{F}$

$$
\mathbf{x}(\mathcal{N})=\frac{1}{N} \sum_{l \in \mathcal{L}} \hat{x}_{l} \mathbf{F}(\mathcal{N}, l)
$$

where $\mathbf{F}(\mathcal{N}, l)=\left(1, W^{-l}, W^{-2 l}, \cdots, W^{-(N-1) l}\right)^{T}$ is the $l$ th column of $\mathbf{F}$. We define $\mathcal{V}_{\mathcal{L}}$ as the subspace that is spanned by the $L$ first columns of $\mathbf{F}$. Consequently

$$
\mathbf{x} \in \mathcal{V}_{\mathcal{L}}=\operatorname{span}\{\mathbf{F}(\mathcal{N}, l)\}_{l \in \mathcal{L}} .
$$




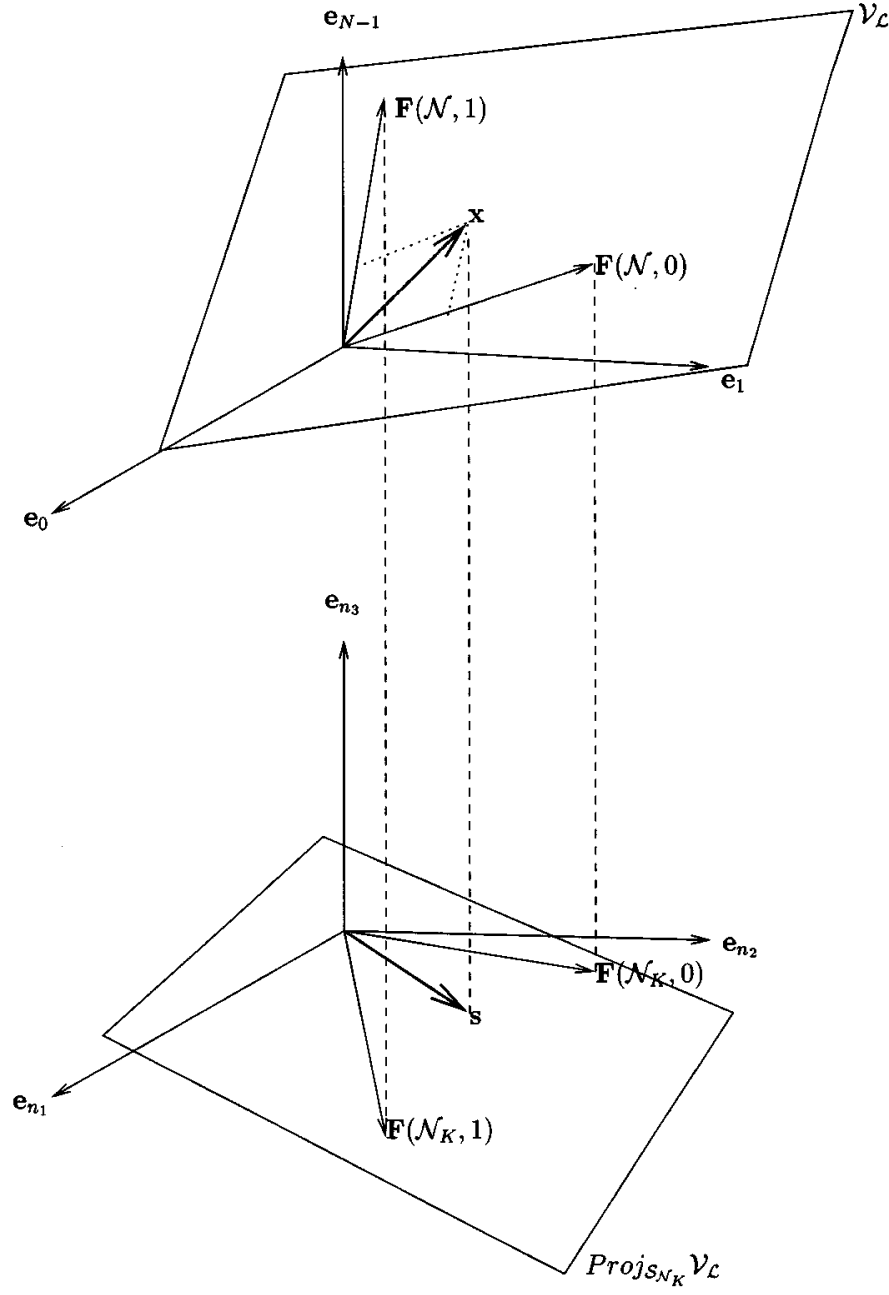

Fig. 2. Geometric interpretation of the ISUL problem for a signal of length $N$ with $K=3$ unknown sample locations and bandlimited to $L=2$. (a) Signal $\mathbf{x}$ belongs to $\mathcal{V}_{\mathcal{L}}$, which is the subspace spanned by the two first columns of the $(\mathbf{D F T})^{-1}$, i.e., $\mathcal{V}_{\mathcal{L}}=\operatorname{span}\left\{\mathbf{F}(\mathcal{N}, 0)=(1,1, \cdots, 1)^{T}, \mathbf{F}(\mathcal{N}, 1)=\right.$ $\left.\left(1, W^{-1}, \cdots, W^{-(N-1)}\right)^{T}\right\}$. (b) Irregular set of sample values $\mathbf{s}$ belonging to the projection of $\mathcal{V}_{\mathcal{L}}$ onto the subspace spanned by $\left\{\mathbf{e}_{n_{1}}, \mathbf{e}_{n_{2}}, \mathbf{e}_{n_{3}}\right\}$, which is denoted by $\mathcal{S}_{\mathcal{N}_{K}}$, where $\mathcal{N}_{K}=\left\{n_{1}, n_{2}, n_{3}\right\}$.

This is illustrated in Fig. 2 for arbitrary $N, K=3$, and $L=2$. Suppose that $\mathcal{N}_{K}=\left\{n_{i}\right\}_{i=1}^{K}$ is an irregular set of locations associated with the sample values $\mathbf{s}$. Then

$$
\mathbf{s}=\frac{1}{N} \mathbf{F}\left(\mathcal{N}_{K}, \mathcal{L}\right) \hat{\mathbf{x}}(\mathcal{L})
$$

and consequently

$$
\begin{aligned}
& \mathbf{s} \in \operatorname{span} \\
& \quad \cdot\left\{\mathbf{F}\left(\mathcal{N}_{K}, l\right)=\left(W^{-n_{1} l}, W^{-n_{2} l}, \cdots, W^{-n_{K} l}\right)^{T}\right\}_{l \in \mathcal{L}} .
\end{aligned}
$$

Define $\mathcal{S}_{\mathcal{N}_{K}}=\operatorname{span}\left\{\mathbf{e}_{n_{i}}\right\}_{i=1}^{K}$ as the subspace spanned by the canonical base vectors associated to the irregular set of locations $\mathcal{N}_{K}$, where $\mathbf{e}_{j}$ is a $N \times 1$ vector, with value 1 in $j$ th position and 0 elsewhere. Then, the $\operatorname{span}\left\{\mathbf{F}\left(\mathcal{N}_{K}, l\right)\right.$ $\left.=\left(W^{-n_{1} l}, W^{-n_{2} l}, \cdots, W^{-n_{K} l}\right)^{T}\right\}_{l \in \mathcal{L}}$ is isomorphic to the projection of $\mathcal{V}_{\mathcal{L}}$ onto $\mathcal{S}_{\mathcal{N}_{K}}$. Thus, we define a solution for the irregular sampling with unknown locations problem as follows:
Definition 2: Suppose $\mathrm{x}$ is a length- $N$ discrete-time $L-B L$ signal. Let $\mathbf{s}$ be a set of $K(K<N)$ sample values, and let $\mathcal{N}_{K}=\left\{n_{i}\right\}_{i=1}^{K}$ be a set of locations. Then, $\mathcal{N}_{K}$ is a solution to the ISUL problem if

$$
\mathrm{s} \in \operatorname{Proj}_{\mathcal{S}_{\mathcal{N}_{K}}} \mathcal{V}_{\mathcal{L}}
$$

The following figures are obvious facts, but we include them to build some intuition on the problem. Fig. 2 gives a geometric interpretation, and Fig. 3 illustrates when a set of locations is and is not a solution to the problem.

We recall that the known variables are $\mathbf{s}, \mathcal{L}, K, N$, and the unknown variables are $\hat{\mathrm{x}}, \mathcal{N}_{K}$. The goal is to determine $\mathrm{x}(\mathcal{N})$. Suppose the location set $\mathcal{N}_{K}$ is known; then, this is achieved by solving the system of $K$ equations and $L$ unknowns $\hat{\mathbf{x}}(\mathcal{L})$ in (6) and then substituting in (3). A least squares solution to the system is obtained by using the generalized inverse ${ }^{1}$ of $\mathbf{F}\left(\mathcal{N}_{K}, \mathcal{L}\right)$, i.e.,

$$
\begin{aligned}
\mathbf{s} & =\mathbf{F}\left(\mathcal{N}_{K}, \mathcal{L}\right) \hat{\mathbf{x}}(\mathcal{L}) \\
\Leftrightarrow \mathbf{F}\left(\mathcal{N}_{K}, \mathcal{L}\right)^{*} \mathbf{s} & =\mathbf{F}\left(\mathcal{N}_{K}, \mathcal{L}\right)^{*} \mathbf{F}\left(\mathcal{N}_{K}, \mathcal{L}\right) \hat{\mathbf{x}}(\mathcal{L}) \\
\Rightarrow \hat{\mathbf{x}}(\mathcal{L}) & =\left[\mathbf{F}\left(\mathcal{N}_{K}, \mathcal{L}\right)^{*} \mathbf{F}\left(\mathcal{N}_{K}, \mathcal{L}\right)\right]^{-1} \mathbf{F}\left(\mathcal{N}_{K}, \mathcal{L}\right)^{*} \mathbf{s}
\end{aligned}
$$

The problem with unknown location set is harder since we obtain a system of $K$ nonlinear equations of the form

$s_{i}=\hat{x}_{0}+\hat{x}_{1} W^{-n_{i}}+\cdots+\hat{x}_{L-1} W^{-n_{i}(L-1)} \quad i=1 \cdots K$

and $L+K$ unknowns $\left\{\hat{x}_{l}\right\}_{l=0}^{L-1},\left\{n_{k}\right\}_{k=1}^{K}$, i.e., more unknown variables than equations.

Note that the spectrum $\hat{\mathrm{x}}(\mathcal{L})$ in $(9)$ depends on the location set $\mathcal{N}_{K}$. If the location set is the right one, then $\mathrm{x}(\mathcal{N})$ is recovered, and we obtain

$$
\mathbf{x}(\mathcal{N})=\mathbf{F}(\mathcal{N}, \mathcal{L})\left[\mathbf{F}\left(\mathcal{N}_{K}, \mathcal{L}\right)^{*} \mathbf{F}\left(\mathcal{N}_{K}, \mathcal{L}\right)\right]^{-1} \mathbf{F}\left(\mathcal{N}_{K}, \mathcal{L}\right)^{*} \mathbf{s}
$$

How can we verify algebraically that a given set of locations $\mathcal{M}_{K}=\left\{m_{i}\right\}_{i=1}^{K}$ is a solution to the ISUL problem? One way is to verify that the sample values at those locations are the same as the observed sample values $\boldsymbol{s}$, i.e.,

$$
\mathrm{x}\left(\mathcal{M}_{K}\right)=\mathbf{s}
$$

or equivalently

$$
\begin{aligned}
& {\left[\mathbf{F}\left(\mathcal{M}_{K}, \mathcal{L}\right)\left[\mathbf{F}\left(\mathcal{M}_{K}, \mathcal{L}\right)^{*} \mathbf{F}\left(\mathcal{M}_{K}, \mathcal{L}\right)\right]^{-1}\right.} \\
& \left.\quad \cdot \mathbf{F}\left(\mathcal{M}_{K}, \mathcal{L}\right)^{*}-\mathbf{I}\right] \mathbf{s}=0 .
\end{aligned}
$$

If (13) is satisfied, then $\mathcal{M}_{K}$ is a solution to the problem.

\section{B. Classification of Solutions}

We want to identify conditions for which there are unique and multiple solutions to the ISUL problem. Intuitively, if the intersection of the subspaces associated with two sets of locations is empty except for $\mathbf{s}=0$, then there is a unique set of locations that correspond to the sample values $\mathbf{s}$.

${ }^{1}$ If $K=L$, then the generalized inverse is the inverse of the matrix. The existence of the generalized inverse or the inverse is assured due to the Vandermonde structure of $\mathbf{F}\left(\mathcal{N}_{K}, \mathcal{L}\right)$. 




(a)

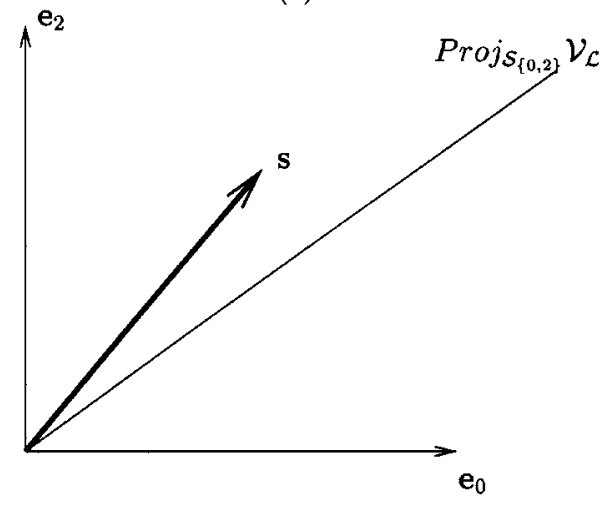

(b)

Fig. 3. (a) Example of a solution $\mathcal{N}_{K}=\{0,1\}$. (b) Example of a nonsolution: $M_{K}=\{0,2\}$.

Theorem 1: A set of locations $\mathcal{N}_{K}$ is a unique solution within shifts to the ISUL problem if

$$
\operatorname{Proj}_{S_{\mathcal{M}_{K}}} \mathcal{V}_{\mathcal{L}} \cap \operatorname{Proj}_{S_{\mathcal{N}_{K}}} \mathcal{V}_{\mathcal{L}}=\emptyset \quad \forall \mathcal{M}_{K} \neq \mathcal{N}_{K}
$$

Proof: For a set of locations $\mathcal{N}_{K}$, the reconstructed signal is given by (11). For another set of locations $\mathcal{M}_{K}$, the reconstructed signal is

$$
\mathrm{x}(\mathcal{N})=\mathbf{F}(\mathcal{N}, \mathcal{L})\left[\mathbf{F}\left(\mathcal{M}_{K}, \mathcal{L}\right)^{*} \mathbf{F}\left(\mathcal{M}_{K}, \mathcal{L}\right)\right]^{-1} \mathbf{F}\left(\mathcal{M}_{K}, \mathcal{L}\right)^{*} \mathbf{s}
$$

Equation (11) is equal to (15) if and only if

$$
\begin{aligned}
& \left(\left[\mathbf{F}\left(\mathcal{N}_{K}, \mathcal{L}\right)^{*} \mathbf{F}\left(\mathcal{N}_{K}, \mathcal{L}\right)\right]^{-1} \mathbf{F}\left(\mathcal{N}_{K}, \mathcal{L}\right)^{*}\right. \\
& \left.\quad-\left[\mathbf{F}\left(\mathcal{M}_{K}, \mathcal{L}\right)^{*} \mathbf{F}\left(\mathcal{M}_{K}, \mathcal{L}\right)\right]^{-1} \mathbf{F}\left(\mathcal{M}_{K}, \mathcal{L}\right)^{*}\right) \mathbf{s}=0 .
\end{aligned}
$$

If there are no common elements in the two subspaces, then the system in (16) is satisfied only if $\mathcal{N}_{K}=\mathcal{M}_{K}$ since $\mathbf{s} \neq \mathbf{0}$.

Similarly, if the intersection of two subspaces generated by different sets of locations is not empty, then there are multiple solutions to the ISUL problem, as illustrated in Fig. 4.

Corollary 1: Given an irregular set of sample values s of an $L-B L$ signal, two sets of locations $\mathcal{M}_{K}$ and $\mathcal{N}_{K}$ are both solutions to the ISUL problem if

$$
\mathbf{s} \in \operatorname{Proj}_{S_{\mathcal{M}_{K}}} \mathcal{V}_{\mathcal{L}} \cap \operatorname{Proj}_{S_{N_{K}}} \mathcal{V}_{\mathcal{L}} \neq \emptyset \quad \forall \mathcal{N}_{K} \neq \mathcal{M}_{K}
$$

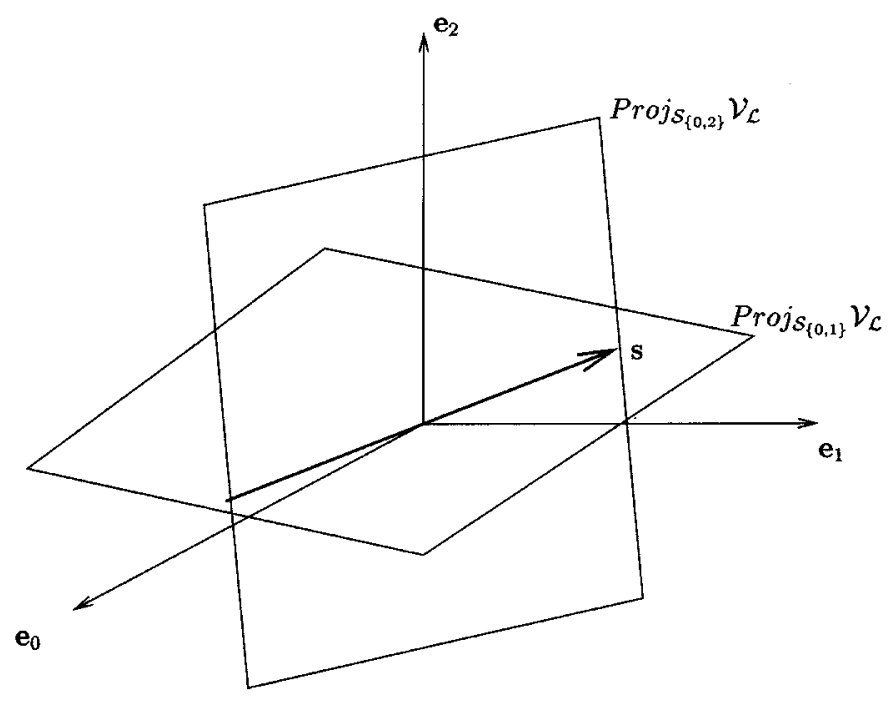

Fig. 4. Example of multiple solutions: $\mathcal{N}_{K}=\{0,1\}, M_{K}=\{0,2\}$. If the sample values $\mathbf{s}$ are such that they lie on the intersection, there is no way to differentiate the sets $\mathcal{N}_{K}$ and $\mathcal{M}_{K}$.

Proof: If the rank of the matrix

$$
\begin{aligned}
& {\left[\mathbf{F}\left(\mathcal{N}_{K}, \mathcal{L}\right)^{*} \mathbf{F}\left(\mathcal{N}_{K}, \mathcal{L}\right)\right]^{-1} \mathbf{F}\left(\mathcal{N}_{K}, \mathcal{L}\right)^{*}} \\
& \quad-\left[\mathbf{F}\left(\mathcal{M}_{K}, \mathcal{L}\right)^{*} \mathbf{F}\left(\mathcal{M}_{K}, \mathcal{L}\right)\right]^{-1} \mathbf{F}\left(\mathcal{M}_{K}, \mathcal{L}\right)^{*}
\end{aligned}
$$

in (16) is greater than $K$, then the system may admit one or more solutions.

For example, if the signal to be reconstructed is $\mathbf{x}=(a, b, a, a, c, a, a, d)^{T}$ and the irregular set of sample values is $\mathbf{s}=(a, b, a, c, a)^{T}$ (i.e., $N=8, K=5$ ), then the possible sets of locations for the sample values are $\mathcal{M}_{K}^{(1)}=\{0,1,2,4,5\}, \mathcal{M}_{K}^{(2)}=\{0,1,2,4,6\}$, $\mathcal{M}_{K}^{(3)}=\{0,1,3,4,5\}$, and $\mathcal{M}_{K}^{(4)}=\{0,1,3,4,6\}$.

Next, we show that if a set of locations is a solution to the ISUL problem, then all shifts of that set is also a solution. This is due to the shift property of the $\mathbf{D F T}_{N}$.

Theorem 2: Let $\mathbf{s}$ be an irregular set of sample values of an $L-B L$ discrete-time signal x. If $\mathcal{N}_{K}=\left\{n_{i}\right\}_{i=1}^{K}$ is a solution to the ISUL problem, then $\mathcal{M}_{K}=\mathcal{N}_{K}+r=\left\{n_{i}+r\right\}_{i=1}^{K}$ with $r \in\left\{1, \cdots, N-n_{K}\right\}$ is also a solution.

Proof: We show that $\operatorname{Proj}_{S_{M_{K}}} \mathcal{V}_{\mathcal{L}}=\operatorname{Proj}_{S_{N_{K}}} \mathcal{V}_{\mathcal{L}}$

$$
\begin{aligned}
\operatorname{Proj}_{\mathcal{M}_{\mathcal{M}_{K}} \mathcal{V}_{\mathcal{L}}} & =\operatorname{span}\left\{\mathbf{F}\left(\mathcal{M}_{K}, l\right)\right\}_{l \in \mathcal{L}} \\
& =\operatorname{span}\left\{\mathbf{F}\left(\mathcal{N}_{K}+r, l\right)\right\}_{l \in \mathcal{L}} \\
& =\operatorname{span}\left\{W_{N}^{r} \mathbf{F}\left(\mathcal{N}_{K}, l\right)\right\}_{l \in \mathcal{L}} \\
& =\operatorname{span}\left\{\mathbf{F}\left(\mathcal{N}_{K}, l\right)\right\}_{l \in \mathcal{L}} \\
& =\operatorname{Proj}_{\mathcal{S}_{\mathcal{N}_{K}}} \mathcal{V}_{\mathcal{L}}
\end{aligned}
$$

Hence, $\mathcal{M}_{K}=\mathcal{N}_{K}+r$ is also a solution to the ISUL problem.

\section{Periodic Nonuniform Sampling with Unknown Shifts}

The periodic nonuniform sampling problem has been studied in [9] and [10] for multiband signals. We consider the ISUL problem where the irregular set of locations follows a periodic nonuniform pattern. It comes about in applications where a uniform set of samples is insufficient to reconstruct the signal, i.e., 
$K<L$. By taking multiple uniform sets differing by a shift, a periodic nonuniform set of locations is formed. The shift is unknown, and therefore, so are the locations. The following example illustrates that by exploiting the periodic structure of the set of locations, the size of the ISUL problem is reduced as well as the computational effort.

Example 1: Consider a discrete-time signal x of length $N=$ 8 bandlimited to $L=4$. Suppose the discrete-time uniform sampling interval is equal to $T=4$; then, the number of samples in the uniform set is $K_{0}=N / T=2<L$, which is insufficient to reconstruct the signal (the number of samples must be at least $L$ ). If we take $C=3$ uniform sets of samples, for example, at locations $\mathcal{N}_{K_{1}}=\{0,4\}, \mathcal{N}_{K_{2}}=\{1,5\}$, and $\mathcal{N}_{K_{3}}=\{2,6\}$, then we obtain a periodic nonuniform set of locations $\mathcal{N}_{K}=$ $\bigcup_{i=1}^{3} \mathcal{N}_{K_{i}}=\{0,1,2,4,5,6\}$. We reformulate the problem by partitioning it according to the periodic structure

$$
\left(\begin{array}{l}
x_{0} \\
x_{4} \\
x_{1} \\
x_{5} \\
x_{2} \\
x_{6}
\end{array}\right)=\frac{1}{8}\left[\begin{array}{cccc}
1 & 1 & 1 & 1 \\
1 & W_{8}^{-4} & 1 & W_{8}^{-4} \\
1 & W_{8}^{-1} & W_{8}^{-2} & W_{8}^{-3} \\
1 & W_{8}^{-5} & W_{8}^{-2} & W_{8}^{-7} \\
1 & W_{8}^{-2} & W_{8}^{-4} & W_{8}^{-6} \\
1 & W_{8}^{-6} & W_{8}^{-4} & W_{8}^{-2}
\end{array}\right] \cdot\left(\begin{array}{c}
\hat{x}_{0} \\
\hat{x}_{1} \\
\hat{x}_{2} \\
\hat{x}_{3}
\end{array}\right) .
$$

We notice that $\left[\begin{array}{cc}1 & 1 \\ 1 & W_{8}^{-4}\end{array}\right]=\left[\begin{array}{cc}1 & 1 \\ 1 & W_{2}^{-1}\end{array}\right]=\mathbf{D F} \mathbf{T}_{2}^{*}$, and breaking it up some more

$$
\begin{aligned}
& 8\left(\begin{array}{l}
\left(\begin{array}{l}
x_{0} \\
x_{4}
\end{array}\right) \\
\left(\begin{array}{l}
x_{1} \\
x_{5}
\end{array}\right) \\
\left(\begin{array}{l}
x_{2} \\
x_{6}
\end{array}\right)
\end{array}\right) \\
& =\left[\begin{array}{cccccc}
\mathrm{DFT}_{2}^{*} & \mathrm{DFT}_{2}^{*} & \mathrm{O}_{2} & \mathrm{O}_{2} & \mathbf{O}_{2} & \mathrm{O}_{2} \\
\mathbf{O}_{2} & \mathbf{O}_{2} & \mathrm{DFT}_{2}^{*} & \mathrm{DFT}_{2}^{*} & \mathbf{O}_{2} & \mathrm{O}_{2} \\
\mathbf{O}_{2} & \mathbf{O}_{2} & \mathrm{O}_{2} & \mathrm{O}_{2} & \mathrm{DFT}_{2}^{*} & \mathrm{DFT}_{2}^{*}
\end{array}\right] \\
& \cdot\left[\begin{array}{cc}
\mathbf{D}_{11} & \mathbf{O}_{2} \\
\mathbf{O}_{2} & \mathbf{D}_{12} \\
\mathbf{D}_{21} & \mathbf{O}_{2} \\
\mathbf{O}_{2} & \mathbf{D}_{22} \\
\mathbf{D}_{31} & \mathbf{O}_{2} \\
\mathbf{O}_{2} & \mathbf{D}_{32}
\end{array}\right] \cdot\left(\begin{array}{l}
\hat{x}_{0} \\
\hat{x}_{1} \\
\hat{x}_{2} \\
\hat{x}_{3}
\end{array}\right)
\end{aligned}
$$

where

$$
\begin{array}{ll}
\mathbf{D}_{11}=\left[\begin{array}{ll}
1 & 0 \\
0 & 1
\end{array}\right], \quad \mathbf{D}_{12}=\left[\begin{array}{ll}
1 & 0 \\
0 & 1
\end{array}\right] \\
\mathbf{D}_{21}=\left[\begin{array}{cc}
1 & 0 \\
0 & W_{8}^{-1}
\end{array}\right], \quad \mathbf{D}_{22}=\left[\begin{array}{cc}
W_{8}^{-2} & 0 \\
0 & W_{8}^{-3}
\end{array}\right] \\
\mathbf{D}_{31}=\left[\begin{array}{cc}
1 & 0 \\
0 & W_{8}^{-2}
\end{array}\right], & \mathbf{D}_{32}=\left[\begin{array}{cc}
W_{8}^{-4} & 0 \\
0 & W_{8}^{-6}
\end{array}\right]
\end{array}
$$

and $\mathbf{O}_{2}$ is a $2 \times 2$ zero matrix. Recall that $\mathbf{D F T}_{2}^{*}=2 \cdot \mathbf{D F T}_{2}^{-1}$; therefore, if we multiply (24) on each side by the matrix

$$
\frac{1}{2}\left[\begin{array}{ccc}
\mathrm{DFT}_{2} & \mathbf{O}_{2} & \mathbf{O}_{2} \\
\mathbf{O}_{2} & \mathrm{DFT}_{2} & \mathbf{O}_{2} \\
\mathbf{O}_{2} & \mathbf{O}_{2} & \mathrm{DFT}_{2}
\end{array}\right]
$$

we obtain the following partitioned system:

$$
4\left(\begin{array}{l}
\mathrm{DFT}_{2} \cdot \mathrm{x}\left(\mathcal{N}_{K_{1}}\right) \\
\mathrm{DFT}_{2} \cdot \mathrm{x}\left(\mathcal{N}_{K_{2}}\right) \\
\mathrm{DFT}_{2} \cdot \mathrm{x}\left(\mathcal{N}_{K_{3}}\right)
\end{array}\right)=\left[\begin{array}{ll}
\mathrm{D}_{11} & \mathrm{D}_{12} \\
\mathrm{D}_{21} & \mathrm{D}_{22} \\
\mathrm{D}_{31} & \mathrm{D}_{32}
\end{array}\right] \cdot\left(\begin{array}{l}
\hat{x}_{0} \\
\hat{x}_{1} \\
\hat{x}_{2} \\
\hat{x}_{3}
\end{array}\right) .
$$

Each block of the partitioned matrix in (26) is a diagonal matrix whose values are given by the rows associated with $x_{0}, x_{1}, x_{2}$ and columns $\{0,1\},\{2,3\}$ of the matrix in (23). The key step in Example 1 that reduces the problem is the multiplication of (24) by the diagonal block DFT $_{2}$ matrix in (25). We generalize by the following:

$$
\begin{gathered}
\frac{N}{K_{0}}\left(\begin{array}{c}
\mathbf{D F T}_{K_{0}} \mathbf{x}\left(\mathcal{N}_{K_{1}}\right) \\
\mathbf{D F T}_{K_{0}} \mathbf{x}\left(\mathcal{N}_{K_{2}}\right) \\
\vdots \\
\mathbf{D F T}_{K_{0}} \mathbf{x}\left(\mathcal{N}_{K_{C}}\right)
\end{array}\right) \\
=\left[\begin{array}{cccc}
\mathbf{D}_{11} & \mathbf{D}_{12} & \cdots & \mathbf{D}_{1 R} \\
\mathbf{D}_{21} & \mathbf{D}_{22} & \cdots & \mathbf{D}_{2 R} \\
\vdots & \vdots & \cdots & \vdots \\
\mathbf{D}_{C 1} & \mathbf{D}_{C 2} & \cdots & \mathbf{D}_{C R}
\end{array}\right] \cdot\left(\begin{array}{c}
\hat{x}_{0} \\
\hat{x}_{1} \\
\vdots \\
\hat{x}_{L-1}
\end{array}\right) \\
\mathbf{y}=\mathbf{D} \cdot \hat{\mathbf{x}}(\mathcal{L})
\end{gathered}
$$

where

$$
\begin{array}{ll}
N & \text { length of the signal; } \\
T & \text { discrete-time uniform sampling interval; } \\
C & \text { number of uniform sets; } \\
K_{0} & \text { size of a uniform set of locations } K_{0}=N / T \in \mathbb{N} ; \\
\mathcal{N}_{K_{i}} & i \text { th uniform set of locations; } \mathcal{N}_{K_{i}}=\{n T+ \\
& \left.r_{i}\right\}_{n=0, \cdots, K_{0}-1, i=1, \cdots, C ;} \\
r_{i} & i \text { th shift from the uniform set }\{n T\}_{n=0}, \cdots, K_{0}-1,0 \leq \\
& r_{i} \leq T-1 ; \\
K & \text { size of the periodic nonuniform set } K=C K_{0} ; \\
L & \text { band limit } L=R K_{0}, 1 \leq R \leq C ; \\
\mathrm{D}_{i j} \quad \begin{array}{l}
\text { diagonal matrices, } \quad \mathrm{D}_{i j}= \\
\end{array} & \left.i \in\left\{(j-1) K_{0}, \cdots, j K_{0}-1\right\}\right), i=1, \cdots, C, j=1, \cdots, R .
\end{array}
$$

To solve the periodic ISUL problem, we must calculate the generalized inverse of the partitioned matrix, which involves matrix multiplication and inverse operations. We obtain a solution in the least squares sense by means of the generalized inverse of the partitioned matrix $\mathbf{D}$ in (27). Hence, we obtain

$$
\hat{\mathbf{x}}(\mathcal{L})=\left(\mathbf{D}^{*} \mathbf{D}\right)^{-1} \mathbf{D}^{*} \mathbf{y}
$$

where $\mathbf{y}=\left[\operatorname{DFT}\left(\mathbf{x}\left(\mathcal{N}_{K_{1}}\right), \cdots, \operatorname{DFT}\left(\mathbf{x}\left(\mathcal{N}_{K_{C}}\right)\right]^{T}\right.\right.$, and $\left(\mathbf{D}^{*} \mathbf{D}\right)^{-1} \mathbf{D}^{*}$ is also a partitioned matrix whose blocks are 




Fig. 5. Fast reconstruction scheme for periodic nonuniform sampling.

diagonal matrices. The fast reconstruction scheme is illustrated in Fig. 5.

1) Computational Complexity: We next compare the computational complexity of the fast reconstruction scheme with the direct unstructured one.

The inverse of a partitioned matrix is obtained from (30) in the Appendix, where we let $\mathbf{A}=\mathbf{D}^{*} \mathbf{D}$. Note that $\mathbf{A}$ is a partitioned $R \times R$ matrix where each block $\mathbf{A}_{m n}$ is a $K_{0} \times K_{0}$ diagonal matrix. Define $o p_{\mathbf{A}}(R)$ as the number of operations required to calculate the inverse of $\mathbf{A}$. We suppose that $R$ is a power of 2 and use a divide-and-conquer approach to determine $\mathbf{A}^{-1}=\left(\mathbf{D}^{*} \mathbf{D}\right)^{-1}$ (i.e., let $\left.\alpha=\{1, \cdots, R / 2\}\right)$. We obtain the following recurrence equation:

$$
\begin{aligned}
o p_{\mathbf{A}}(R) & =10 o p_{\mathbf{A}}(R / 2)+12(R / 2)^{3} K_{0}+4(R / 2)^{2} K_{0} \\
o p_{\mathbf{A}}(1) & =K_{0}
\end{aligned}
$$

and conclude that $o p_{\mathbf{A}}(R) \in O\left(R^{\log _{2} 10} K_{0}\right)$. Although $\log _{2} 10$ is larger than 3 , the complexity of our fast scheme is by far still less than the direct scheme because $R$ is much less than $L$. We summarize the number of operations (matrix multiplication, matrix inversion) required for each scheme in Table I. Furthermore, by substituting $K=C K_{0}$ and $L=R K_{0}$ in Table I, keeping the variables $C$ and $R$ constant, and letting $K_{0}$ vary, the computational complexities of the two schemes are compared in Fig. 12.

\section{NumericAl SOLVING Methods}

We numerically solve the ISUL problem by considering it as a combinatorial optimization problem. We describe three solving methods. We test the optimality of a solution $\mathcal{M}_{K}$ by verifying if the $\ell^{2}$-norm of (13)

$$
\begin{gathered}
E\left(\mathcal{M}_{K}\right)=\|\left[\mathbf{F}\left(\mathcal{M}_{K}, \mathcal{L}\right)\left[\mathbf{F}\left(\mathcal{M}_{K}, \mathcal{L}\right)^{*} \mathbf{F}\left(\mathcal{M}_{K}, \mathcal{L}\right)\right]^{-1}\right. \\
\left.\cdot \mathbf{F}\left(\mathcal{M}_{K}, \mathcal{L}\right)^{*}-\mathbf{I}\right] \mathbf{s} \|
\end{gathered}
$$

equals zero for the first method, which is an exhaustive search method. The other two methods (the cyclic coordinate and random search methods) are both descent heuristics that minimize $E\left(\mathcal{M}_{K}\right)$. The main goal of this paper is to find a solution to the ISUL problem; therefore, we used the direct method to calculate $E\left(\mathcal{M}_{K}\right)$, which may be computationally expensive for large problems. We can further reduce the computations by noting that the matrix $\mathbf{F}^{*} \mathbf{F}$ is a Toeplitz matrix and can be obtained by computing only the first row, i.e., $K L$ operations. Consequently, by fast Toeplitz methods, it can be inverted in $C L, \log L$ operations [3].
TABLE I

SUMMARY OF COMPLEXITY O(operations) FOR BOTH SCHEMES

\begin{tabular}{c|c||c|c}
\hline \multicolumn{4}{c}{ Complexity } \\
\hline \multicolumn{3}{c}{ Direct scheme } & \multicolumn{2}{c}{ Fast scheme } \\
\hline $\mathbf{F}^{*} \mathbf{F}$ & $L^{2} K$ & $\mathbf{D}^{*} \mathbf{D}$ & $R^{2} C K_{0}$ \\
\hline$\left(\mathbf{F}^{*} \mathbf{F}\right)^{-1}$ & $L^{3}$ & $\left(\mathbf{D}^{*} \mathbf{D}\right)^{-1}$ & $R^{\log _{2} 10} K_{0}$ \\
\hline $\mathbf{F}^{*} \cdot \mathbf{x}\left(\mathcal{N}_{K}\right)$ & $L K$ & $\mathbf{D}^{*} \mathbf{y}$ & $R C K_{0} \log K_{0}$ \\
\hline
\end{tabular}

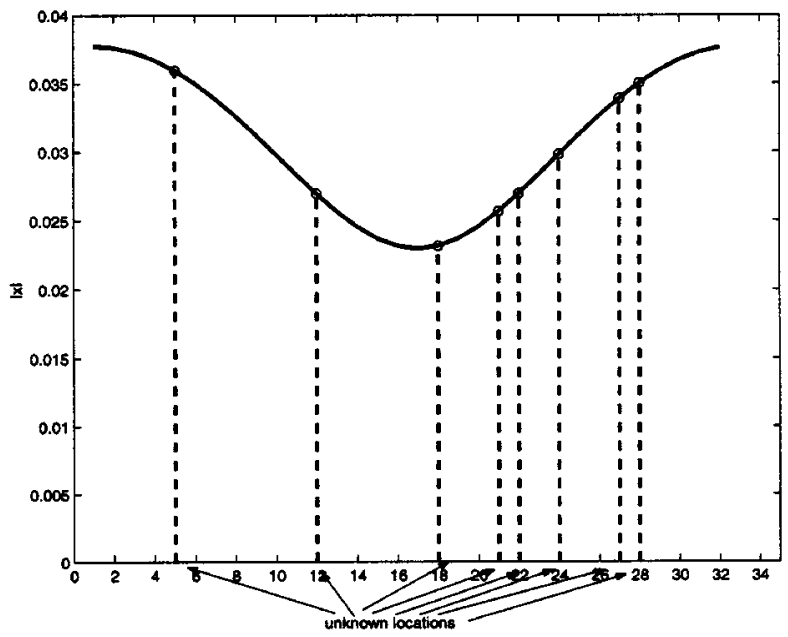

Fig. 6. Discrete-time signal of length $N=32$ with a random set of unknown locations of size $K=8$.

\section{A. Exhaustive Method}

An elementary way to solve the ISUL problem is using an exhaustive search approach. This method consists of verifying (29) for all $\left(\begin{array}{c}N \\ K\end{array}\right)$ sets of locations. From Theorem 2, since some sets of locations are just shifts of each other, we put these sets in one class and elect a representative for each class. The representative satisfying (29) with $E\left(\mathcal{M}_{K}\right)=0$ is a solution of the ISUL problem. Clearly, this method can only be used for small size problems because of the combinatorial explosion, and multiple solutions are identified on such type of problems. We can construct them algebraically, but these are extremely unlikely to be found in practice.

\section{B. Descent Methods: Random Search and Cyclic Coordinate}

The random search method is an iterative descent algorithm. A component of the location set is perturbed according to a probability distribution. If the cost function $\left(E\left(\mathcal{M}_{K}\right)\right)$ of the perturbed solution decreases, we keep perturbing the same component. Otherwise, we perturb another component and continue this until a global or local minimum is found.

\footnotetext{
Algorithm 1: Random search method

1) Initialization:

Choose an initial set of $K$ locations

$\mathcal{M}_{K}^{(1)}$, and let $\mathcal{M}_{K}=\mathcal{M}_{K}^{(1)}, j=1$.

Go to General step.

2) General step:
} 


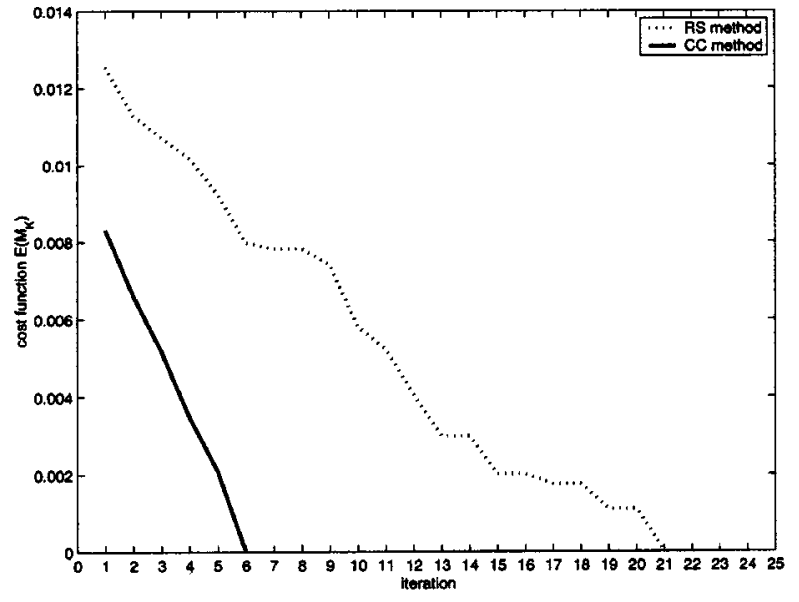

(a)

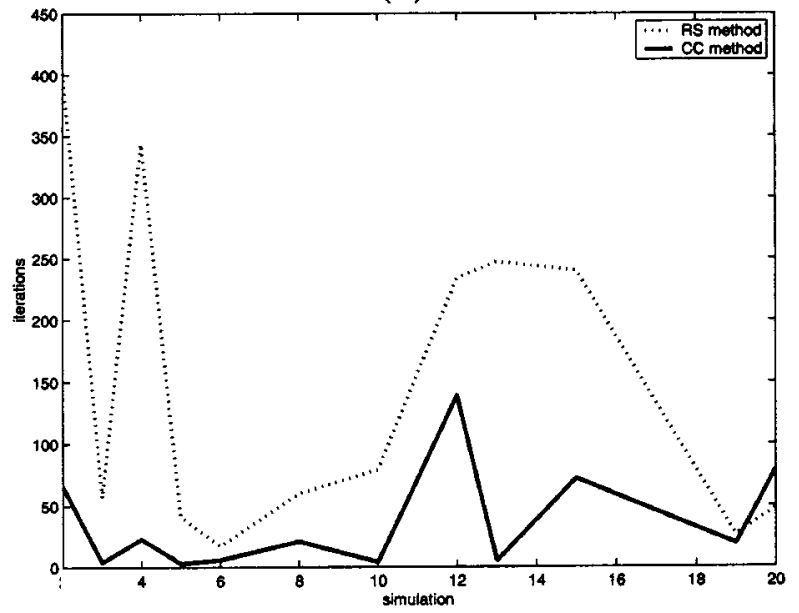

(b)

Fig. 7. (a) Convergence of the cyclic coordinate (CC) and random search (RS) method on a discrete-time signal of length $N=32$ with a random set of unknown locations of size $K=8$ and bandlimited to $L=2$. Here, the global minimum has been found in the first attempt. (b) Total number of iterations to find a global minimum when multiple local minima are encountered. The maximum number of local minima was set to 30 .

a) Obtain $\mathcal{M}_{K}^{(2)}$ by the perturbing component $j$ of $\mathcal{M}_{K}$ by $\lambda$, where $\lambda \sim \operatorname{Bin}(2 M, p)$.

b) If $E\left(\mathcal{M}_{K}^{(2)}\right)=0$, then $\mathcal{M}_{K}=\mathcal{M}_{K}^{(2)}$ is a global minimum. Stop.

c) If $\left|E\left(\mathcal{M}_{K}^{(2)}\right)-E\left(\mathcal{M}_{K}\right)\right|=0$, then $\mathcal{M}_{K}$ is a local minimum. ${ }^{2}$

d) If $E\left(\mathcal{M}_{K}^{(2)}\right)<E\left(\mathcal{M}_{K}^{(1)}\right)$, then the current set $\mathcal{M}_{K}=\mathcal{M}_{K}^{(2)}, \mathcal{M}_{K}^{(1)}=\mathcal{M}_{K}^{(2)}$.

e) Let $j=j+1, \mathcal{M}_{K}=\mathcal{M}_{K}^{(1)}$. If $j \leq K$, repeat step $2 \mathrm{a}$

The cyclic coordinate method [11] is similar to the gradient descent in that it descends in the best direction but does not require any derivative information. It uses the coordinate direction axis as the search directions. It differs from the Random search

${ }^{2}$ There is a local minimum when the cost function value remains the same from one perturbation to another.

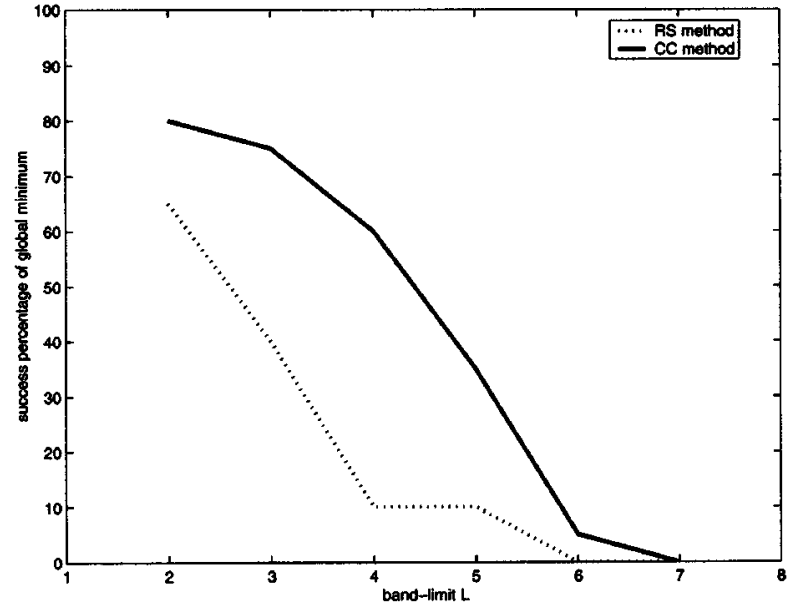

Fig. 8. Success percentage of finding global minimum using the $\mathrm{CC}$ and RS methods for signals of length $N=32$ with a random sampling set of $K=8$ locations. Average of 20 simulations on 20 different signals.

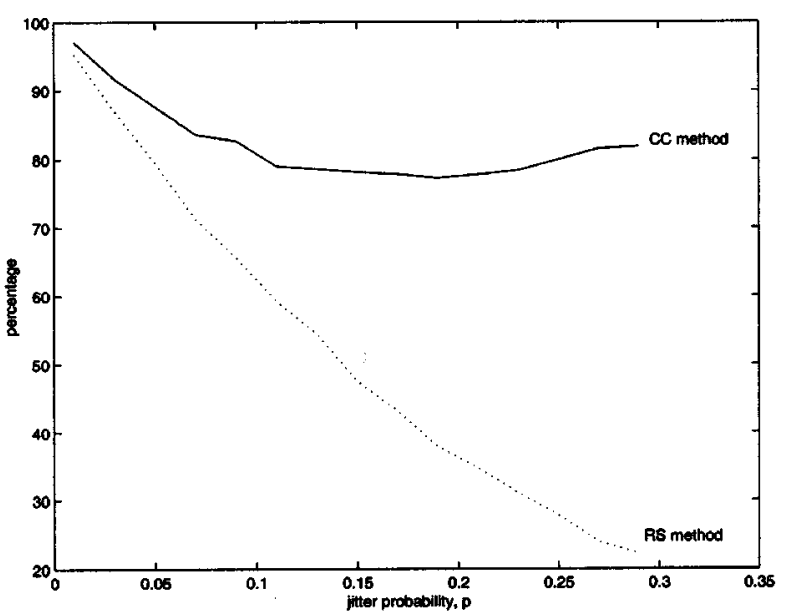

Fig. 9. Success percentage of finding global minimum using $\mathrm{CC}$ and RS methods for sampling with jitter in locations where the jitter follows a binomial probability distribution with parameters $(2, p)$ centered at $\epsilon=0$. Average of 100 simulations on 50 different signals with parameters $N=16, K=4, L=2$.

method in that the perturbation is not probabilistic but deterministic.

\section{Algorithm 2: Cyclic coordinate method}

1) Initialization:

Let $\mathbf{d}_{1}, \cdots, \mathbf{d}_{K}$ be the coordinate directions, where $\mathrm{d}_{i}$ is $1 \times K$ vector with value 1 in position

$i$ and 0 elsewhere. Choose an initial set of $K$ locations $\mathcal{M}_{K}^{(1)}$, and let $\mathcal{M}_{K}=\mathcal{M}_{K}^{(1)}$, $j=1$.

Go to General step.

2) General Step:

a) $\mathcal{M}_{K}$ is obtained by perturbing component $j$ of $\mathcal{M}_{K}^{(1)}$ by $\lambda_{j}=\operatorname{argmin}-M \leq \lambda \leq M$ $\left\{E\left(\mathcal{M}_{K}^{(1)}+\lambda \mathbf{d}_{j}\right)\right\}$. Let $\mathcal{M}_{K}=\mathcal{M}_{K}^{(1)}+\lambda_{j} \mathbf{d}_{j}$.

b) If $E\left(\mathcal{M}_{K}\right)=0$, then $\mathcal{M}_{K}$ is a global minimum. Stop. 


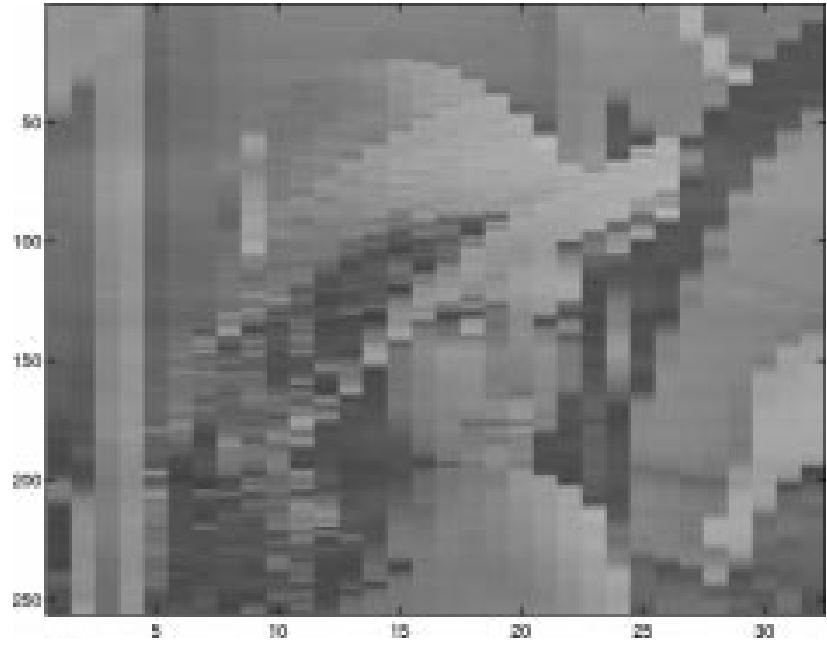

(a)



(b)

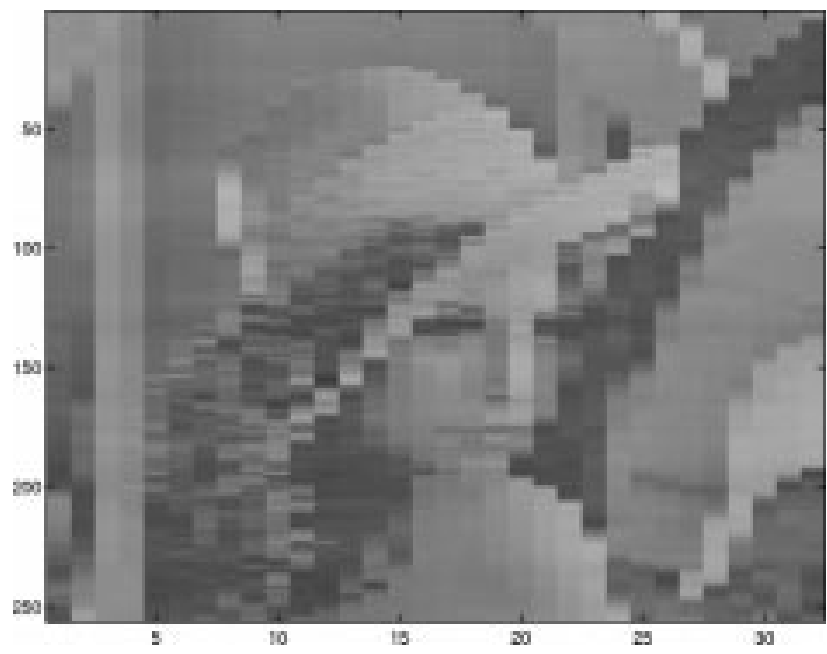

(c)

Fig. 10. Three copies $(C=3)$ of Lena with low resolution. Each image is of size $N \times K_{0}, N=256, K_{0}=32$.

c) If $\left|E\left(\mathcal{M}_{K}^{(1)}\right)-E\left(\mathcal{M}_{K}\right)\right|=0$, then $\mathcal{M}_{K}$ is a local minimum.

d) Let $j=j+1, \mathcal{M}_{K}^{(1)}=\mathcal{M}_{K}$. If $j \leq K$, then repeat step 2 a.
If $j>K$, then let $j=1$, and repeat step $2 \mathrm{a}$.

Both methods do not guarantee a global minimum. When stuck in a local minimum, a random set of locations is generated, and the methods are repeated. The tested algorithm comes to a halt when a global minimum is attained or an upper bound on the number of local minima is exceeded.

\section{EXPERIMENTAL RESULTS}

In this section, we apply the methods described in Section III to find three types of irregular sets:

1) random set of locations;

2) jittered set of locations;

3) periodic nonuniform set of locations.

\section{A. Unknown Random Sampling Set of Locations}

In this experiment, we want to find the locations of a random sampled discrete-time signal of length $N=32$ with $K=8$ random samples and bandlimited to $L=2$; see Fig. 6. In Fig. 7(a), we compare the convergence of the $\mathrm{CC}$ and RS methods and conclude that the CC method converges faster. This is due to the deterministic nature of the algorithm. We also illustrate how the number of iterations required to find a global minimum varies from one simulation to another in Fig. 7(b). We restricted the maximum number of local minima to 30. Finally, in Fig. 8, we show that the probability of finding a global minimum depends on the smoothness of a signal, i.e., the smaller the band limit $L$ as compared with $K$, the more likely it is to find the right set of locations.

\section{B. Unknown Jittered Sampling Set of Locations}

In this experiment, we take samples around multiples of a sampling interval but with a certain time location uncertainty. Formally, the irregular set of locations is $\mathcal{N}_{K}=\left\{n T+\epsilon_{n}\right\}_{n=0}^{K-1}$, where $\epsilon$ is the jitter. We assume the jitter follows a binomial distribution centered at $\epsilon=0$ with parameters $(2, p)$, where $p$ is the probability of no jitter, and $\epsilon \in\{-1,0,1\}$. We applied the RS and CC methods on jittered data with $p$ varying from 0.01 to 0.3 . Fig. 9 compares the two methods, and we conclude that the percentage of finding a global minimum using the $\mathrm{CC}$ method is on average $83 \%$.

\section{Periodic Nonuniform Sampling Set with Unknown Shifts}

We apply the exhaustive method to solve the periodical ISUL. The descent methods described in Section III-B are not appropriate since they do not consider the nice structure of the problem. Thanks to our fast scheme, the number of possible locations is reduced to $\left(\begin{array}{l}T \\ C\end{array}\right) \ll\left(\begin{array}{l}N \\ K\end{array}\right)$.

1) Application-Super-resolution: One application of the periodic nonuniform sampling with unknown shifts is for instance if we want to improve the resolution of an image by taking several samplings of the same image but with a shift. For simplicity, we suppose that the spectrum is such that the first $L$ columns of the 2-D DFT are nonzero and that the last $N-L$ columns are zeros. Therefore, the 2-D case is simply an extension of the 1-D case. Hence, we need only find the 




(a)

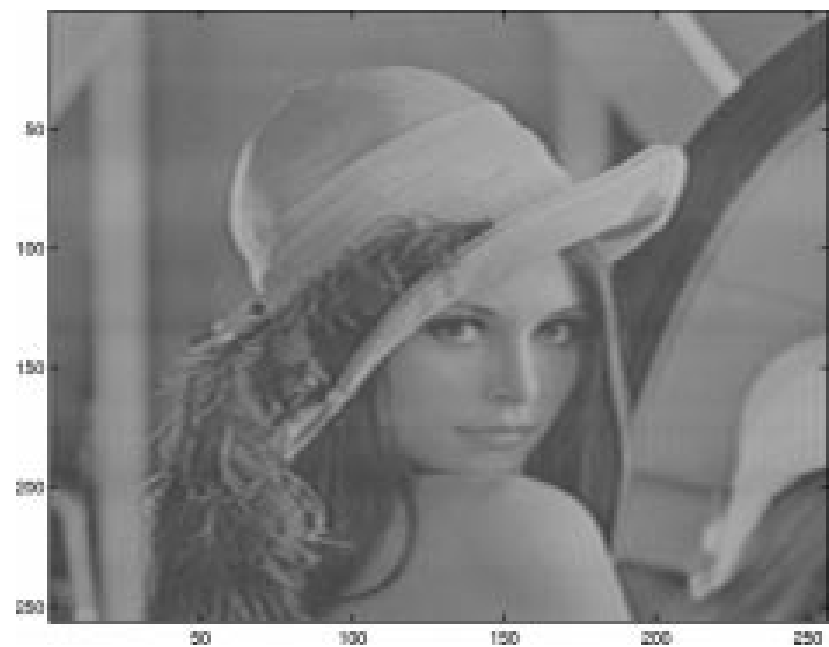

(b)

Fig. 11. (a) Periodic nonuniform sampling set obtained from three copies of Lena put on a finer grid $\left(N \times K_{0} \cdot T, N=256, K_{0}=32, T=8\right)$ according to the shifts found by the exhaustive method $\mathcal{N}_{K_{i}}=\left\{r_{i}+n T\right\}_{n_{0}}^{K_{0}-1}, r=$ $(1,3,5), i=1, \cdots, C$. (b) Reconstruction of Lena.

shifts on one row of the image and suppose that they are the same for the other rows. Fig. 10 shows three images of Lena of size $256 \times 32$. Each of the three images were taken from a $256 \times 256$ image and differ by a horizontal shift. The discrete uniform sampling period is $T=8$, and therefore, the shifts must be between 0 and 7. Using the exhaustive method instead of verifying $\left(\begin{array}{c}256 \\ 3 \times 32\end{array}\right)$ locations, we are able to determine the shifts by verifying $\left(\begin{array}{l}T \\ C\end{array}\right)=\left(\begin{array}{l}8 \\ 3\end{array}\right)$ locations. Once the shifts are found, we place each set of data on a finer grid of size $256 \times 256$ and reconstruct the image. The periodic nonuniform sampled image and the reconstruction are shown in Fig. 11.

\section{CONCLUSION}

We have studied the irregular sampling with unknown locations problem for discrete-time bandlimited signals. We have derived a solution and showed, in certain cases, the existence of multiple solutions. We have described three methods that numerically solve the ISUL problem. We have shown that for the

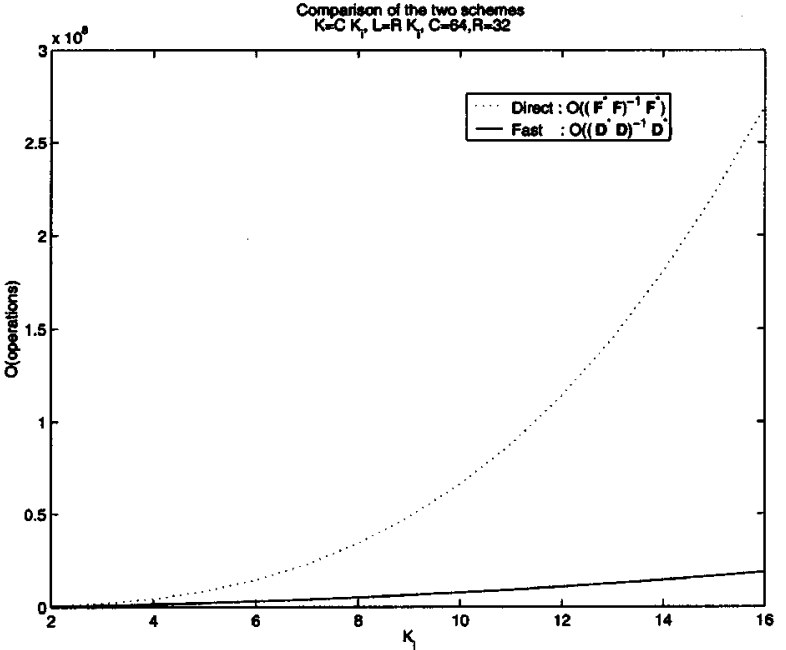

Fig. 12. Comparison of the unstructured direct method and the fast structured scheme.

periodic nonuniform case, the computational complexity of the problem can be substantially reduced. In particular, we have developed a fast method to calculate the generalized inverse by exploiting the periodic structure of the set of locations in the DFT matrix. We have shown how we can apply this to improve the resolution of an image. As future work, we are investigating the unknown locations problem in two dimensions, as well as improving the search methods. One promising method is the Tabu search [13], which keeps a tabu list, i.e., a list of bad perturbations, and avoids getting stuck in local minima.

\section{APPENDIX}

\section{A. Inverse of a Partitioned Matrix}

Suppose $\mathbf{A}$ is an $R \times R$ partitioned matrix

$$
\mathbf{A}=\left[\begin{array}{cccc}
\mathbf{A}_{11} & \mathbf{A}_{12} & \cdots & \mathbf{A}_{1 R} \\
\mathbf{A}_{21} & \mathbf{A}_{22} & \cdots & \mathbf{A}_{2 R} \\
\vdots & \vdots & \cdots & \vdots \\
\mathbf{A}_{R 1} & \mathbf{A}_{R 2} & \cdots & \mathbf{A}_{R R}
\end{array}\right]
$$

where $\mathbf{A}_{m n}$ are square matrices. The inverse $\mathbf{B}=\mathbf{A}^{-1}$ of a partitioned matrix can be found in [8] by

$$
\begin{aligned}
\mathbf{B}(\alpha, \alpha)= & {\left[\mathbf{A}(\alpha, \alpha)-\mathbf{A}\left(\alpha, \alpha^{\prime}\right) \mathbf{A}\left(\alpha^{\prime}, \alpha^{\prime}\right)^{-1} \mathbf{A}\left(\alpha^{\prime}, \alpha\right)\right]^{-1} } \\
\mathbf{B}\left(\alpha, \alpha^{\prime}\right)= & \mathbf{A}(\alpha, \alpha)^{-1} \mathbf{A}\left(\alpha, \alpha^{\prime}\right) \\
& \cdot\left[\mathbf{A}\left(\alpha^{\prime}, \alpha\right) \mathbf{A}(\alpha, \alpha)^{-1} \mathbf{A}\left(\alpha, \alpha^{\prime}\right) \mathbf{A}\left(\alpha^{\prime}, \alpha^{\prime}\right)\right]^{-1}
\end{aligned}
$$

where $\alpha$ is a subset of $\{1, \cdots, R\}$, and $\alpha^{\prime}$ is the complement index set of $\alpha$. For instance, if $R=4$ and $\alpha=\{1,2\}, \alpha^{\prime}=\{3,4\}$, then $\mathbf{A}(\alpha, \alpha)=\left[\begin{array}{ll}\mathbf{A}_{11} & \mathbf{A}_{12} \\ \mathbf{A}_{21} & \mathbf{A}_{22}\end{array}\right]$, and $\mathbf{A}\left(\alpha, \alpha^{\prime}\right)=\left[\begin{array}{ll}\mathbf{A}_{13} & \mathbf{A}_{14} \\ \mathbf{A}_{23} & \mathbf{A}_{24}\end{array}\right]$. 


\section{ACKNOWLEDGMENT}

The authors would like to thank M. Gastpar and P. Prandoni for feedback. They would especially like to thank F. Marvasti for his stimulating questions and R. Pribic and J. Vieira for the interesting discussions during the Sampling Theory and Applications' 99 workshop. The authors would also like to acknowledge the reviewers for their helpful comments.

\section{REFERENCES}

[1] R. M. Young, An Introduction to Nonharmonic Fourier Series. New York: Academic, 1980.

[2] H. G. Feichtinger and K. Gröchenig, "Theory and practice of irregular sampling," in Wavelets: Mathematics and Applications, J. J. Benedetto and M. W. Frazier, Eds. Boca Raton, FL: CRC, 1994, pp. 305-363.

[3] H. G. Feichtinger, K. Gröchenig, and T. Strohmer, "Efficient numerical methods in nonuniform sampling theory," Numer. Math., vol. 69, pp. 423-440, 1995.

[4] K. Gröchenig, "A discrete theory of irregular sampling," Lin. Algebra Appl., vol. 193, pp. 129-150, 1993.

[5] T. Strohmer, "Computationally attractive reconstruction of bandlimited images from irregular samples," IEEE Trans. Image Processing, vol. 6, pp. 540-548, June 1997.

[6] P. Marziliano and M. Vetterli, "Irregular sampling with unknown locations," in Proc. IEEE Int. Conf. Acoust., Speech, Signal Process., Phoenix, AZ, Mar. 1999.

[7] P. J. S. G. Ferreira and J. M. N. Vieira, "Detection and correction of missing samples," in Proc. Workshop Sampling Theory Appl., Aveiro, Portugal, June 1997, pp. 169-174.

[8] R. A. Horn and C. R. Johnson, Matrix Analysis. New York: Cambridge Univ. Press, 1985

[9] B. Foster and C. Herley, "Exact reconstruction from periodic nonuniform samples," in Proc. IEEE Int. Conf. Acoust., Speech, Signal Process., vol. 5, Detroit, MI, May 1995, pp. 1452-1455.

[10] P. Feng and Y. Bresler, "Spectrum-blind minimum-rate sampling and reconstruction of multi-band signals," in Proc. IEEE Int. Conf. Acoust., Speech, Signal Process., vol. 3, Atlanta, GA, May 1996, pp. 1688-1691.

[11] H. D. Sherali, M. S. Bazaraa, and C. M. Shetty, Nonlinear Programming Theory and Algorithms. New York: Wiley, 1993.

[12] R. J. Marks II, Advanced Topics in Shannon Sampling and Interpolation Theory. Seattle, WA: Springer-Verlag, 1992.

[13] F. Glover, "Tabu search-Part I," ORSA J. Comput., vol. 1, no. 3, pp 190-206, Summer 1989.

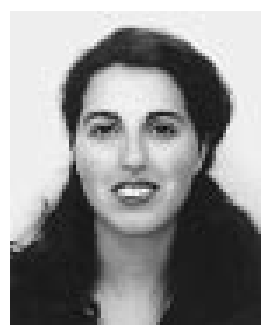

Pina Marziliano was born in Montreal, PQ, Canada, in 1971. She received the B.Sc. degree in applied mathematics and the M.Sc. degree in computer science, specializing in operations research, from the Université de Montréal in 1994 and 1996, respectively. She is currently pursuing the Ph.D. degree at the Audio Visual Communications Laboratory, Communication Systems Department, Swiss Federal Institute of Technology, Lausanne, Switzerland.

She received two summer internship scholarships (in 1992 and 1993) as well as a grant for the M.Sc. degree from the Natural Sciences and Engineering Research Council of Canada (NSERC). Her current research interests are sampling theory and applications in communications and signal processing.

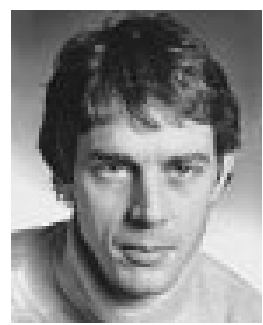

Martin Vetterli (F'95) received the Dipl.El.-Ing. degree from ETH Zürich (ETHZ), Zürich, Switzerland, in 1981, the M.S. degree from Stanford University, Stanford, CA, in 1982, and the D.Sc. degree from EPF Lausanne (EPFL), Lausanne, Switzerland, in 1986.

He was a Research Assistant at Stanford and EPFL and has worked for Siemens and AT\&T Bell Laboratories. In 1986, he joined Columbia University, New York, NY, where he was an Associate Professor of Electrical Engineering and Co-Director of the Image and Advanced Television Laboratory. In 1993, he joined the University of California, Berkeley, where he was a Professor with the Department of Electrical Engineering and Computer Sciences until 1997. He now holds an Adjunct Professor position. Since 1995, he has been a Professor of Communication Systems at EPFL, where he chaired the Communications Systems Division from 1996 to 1997, and heads the Audio-Visual Communications Laboratory. He held visiting positions at ETHZ in 1990 and at Stanford in 1998. His research interests include wavelets, multirate signal processing, computational complexity, signal processing for telecommunications, digital video processing and compression, and wireless video communications. He is on the editorial boards of Annals of Telecommunications, Applied and Computational Harmonic Analysis, and The Journal of Fourier Analysis and Applications. He is the co-author, with J. Kovacević, of the book Wavelets and Subband Coding (Englewood Cliffs, NJ: Prentice-Hall, 1995). He has published about 85 journal papers on a variety of topics in signal and image processing and holds five patents.

Dr. Vetterli is a member of SIAM and was the Area Editor for Speech, Image, Video, and Signal Processing of the IEEE TRANSACTIONS ON COMMUNICATIONS. He received the Best Paper Award of EURASIP in 1984 for his paper on multidimensional subband coding, the Research Prize of the Brown Bovery Corporation (Switzerland) in 1986 for his doctoral thesis, the IEEE Signal Processing Society's Senior Award in 1991 and in 1996 (for papers with D. LeGall and K. Ramchandran, respectively), and was a IEEE Signal Processing Distinguished Lecturer in 1999. He received the Swiss National Latsis Prize in 1996 and the SPIE Presidential award in 1999. He is a member of the Swiss Council on Science and Technology. He was a plenary speaker at various conferences (e.g., 1992 IEEE ICASSP). 